CLINICAL ALERT

\title{
Varicose veins: Look before you strip - the occluded inferior vena cava and other lurking pathologies
}

\author{
T Mokoena \\ Prof. Taole Mokoena, MB ChB, DPhil, FRCS, is the Head of Surgery at the School of Medicine, University of Pretoria, South Africa, and the Pretoria \\ Academic Hospital Complex. He is a keen observer of disease and has a wide-ranging interest in surgery.
}

Corresponding author: T Mokoena (taole.mokoena@up.ac.za)

Lower limb varicose veins are a common complication of bipedal human movement and deep-vein thrombosis. However, they may have unusual causes, e.g. forming as collaterals around an obstruction or resulting from vascular malformations. Surgery in these cases can be inappropriate or harmful. Five cases of lower limb varicose veins in which there was underlying pathology highlight the fact that cursory examination of patients with varicose veins and inappropriate special investigations can miss rare but significant underlying pathology. Patients should be examined systematically, and varicose veins in unusual situations should alert the clinician. Inappropriate surgery can be harmful.

S Afr Med J 2014;104(10):668-670. DOI:10.7196/SAMJ.8395

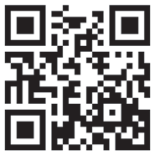

There are many causes of dilated tortuous subcutaneous veins. The common varicose veins of the legs result from excessive blood flow due to incompetence of unidirectional valve systems. The latter are either primarily defective or overwhelmed by gravitational force in the prolonged upright position. The flow of blood in such veins is in the natural direction. Dilated veins may also be collaterals that allow flow of blood around an obstruction, such as the oesophageal collaterals in portal hypertension. Collaterals can also develop in the limbs when deep veins are occluded. Depending on the anatomical location, flow in these collaterals may be reversed.

Classic varicose vein surgery on the legs is aimed at removing the abnormal veins by stripping and addressing the incompetent flow channels by performing vein ligation. Surgery on collaterals around a venous obstruction may be inappropriate and even harmful, and surgery on varicose veins caused by arteriovenous (AV) malformations is also inappropriate. Five cases in which varicose vein surgery had been inappropriately performed or requested are presented. These situations could have been prevented by thorough clinical examination and consequent appropriate imaging studies.

\section{Case reports}

\section{Case 1}

A 41-year-old man was referred to the surgical outpatient department at Kalafong Hospital, Pretoria, South Africa, for management of an indolent leg ulcer and 'lower limb' varicose veins. On clinical examination, both legs and feet showed dystrophic skin changes with ulceration medially above the right ankle. There were varicose veins in both lower limbs, more marked on the right, and tortuous distended superficial inferior and superior epigastric veins on the trunk. The direction of flow in the latter was cephalad. The primary doctor had established patency and competence of the deep veins of the leg by Doppler studies and had referred the patient for vein stripping and ligation.

In view of the varicose veins on the trunk, further imaging was undertaken. This revealed occlusion of the inferior vena cava (IVC). No surgery was performed.

\section{Case 2}

A 46-year-old man presented with bilateral leg ulcers and unilateral lower limb varicose veins. Varicose vein stripping had been performed 2 years previously for varicose ulcers in one leg. Examination of the abdominal wall revealed varicosity of the superficial inferior epigastric veins arising at the groin, with cephalad flow. Venography confirmed IVC occlusion. He was treated non-operatively with local wound care and bilateral full-leg compression stockings.

\section{Case 3}

A 12-year-old boy presented with a longstanding lump over the right groin which became prominent and painful on straining. The referring practitioner considered it to be an inguinal hernia. However, on examination of the patient's trunk it soon became evident that the lump was the distal extent of an abdominothoracic varicose channel arising from the right groin. A duplex Doppler scan of the lower limb and IVC failed to reveal the suspected venous occlusion. It was later demonstrated in the distal IVC by transfemoral venography, which also showed ascending lumbar vein collaterals draining into the proximal IVC. The cause of the occlusion was unknown, and no surgery was performed.

\section{Case 4}

A 42-year-old female nurse presented with recurrent varicose veins after previous bilateral saphenous vein stripping performed elsewhere. On close examination, tortuous venous channels on the torso with femoroaxillary drainage were noted. Imaging of the IVC by venography revealed retrohepatic IVC occlusion. Further investigations confirmed a liver tumour in segment IV, which was confirmed to be a fibrolamellar hepatoma. It was managed by transarterial chemoembolisation, and a follow-up computed tomography scan of the liver showed that it has shrunk completely. No further venous surgery was performed.

\section{Case 5}

A 29-year-old man with a lifelong history of varicosities of the left lower limb presented with painful swelling of that leg. He had 
undergone surgery on the same leg 12 years earlier for removal of varicose veins. He had varicosities on the lateral and posterior aspects of both lower limbs as well as the gluteal region. There were dystrophic skin changes on the legs but no ulcers. There were no bruits over the varices. Arteriography showed extensive AV malformations of the popliteal, anterior and posterior tibial and peroneal vessels. The patient was managed with compression stockings. Fig. 1 depicts a similar case in which the AV malformation was unilateral. The patient first presented to us, was correctly diagnosed, and was managed similarly.

\section{Discussion}

Thrombosis of the deep veins of the lower limb and destruction of their valve systems is a well-recognised cause of varicose veins and their sequelae of dystrophic skin changes and ulceration. ${ }^{[1]}$ That these varicosities can also arise from 'reverse' venous flow from IVC obstruction and resultant saphenofemoral incompetence requires emphasis. Cursory physical examination and inappropriately directed imaging in such cases may mean that underlying pathology is overlooked or misdiagnosed. The concern is that with more widespread use of modern technology such as ultrasound scanning in clinical medicine, long-established teaching and simple clinical tests are increasingly being ignored or unlearned. ${ }^{[2]}$ This is particularly pertinent to primary family doctors, whose advice their patients tend, understandably, to trust more than that of an unknown specialist.

There is a growing tendency for patients to resist being subjected to a full physical examination, apart from their perceived area of pathology. ${ }^{[3]}$ Reluctance in this regard on the part of doctors has also increased, especially in view of the increase in charges of improper conduct. However, clinical examination of the torso could reveal varicosities as depicted in Fig. 2, and alert the clinician to possible major intra-abdominal venous occlusion. In the five cases described above, the tortuous trunk vessels with reverse flow could be discerned clinically and should have prompted further investigation for occlusion of the IVC. If Doppler studies alone, often done by technical staff and in the absence of a thorough clinical assessment, are used to study deep limb veins, there is a risk that saphenous varicosities will be incorrectly attributed to saphenofemoral venous incompetence.

The most common cause of occlusion of the major veins in the abdomen is thrombosis. ${ }^{[4]}$ The patients described here presented

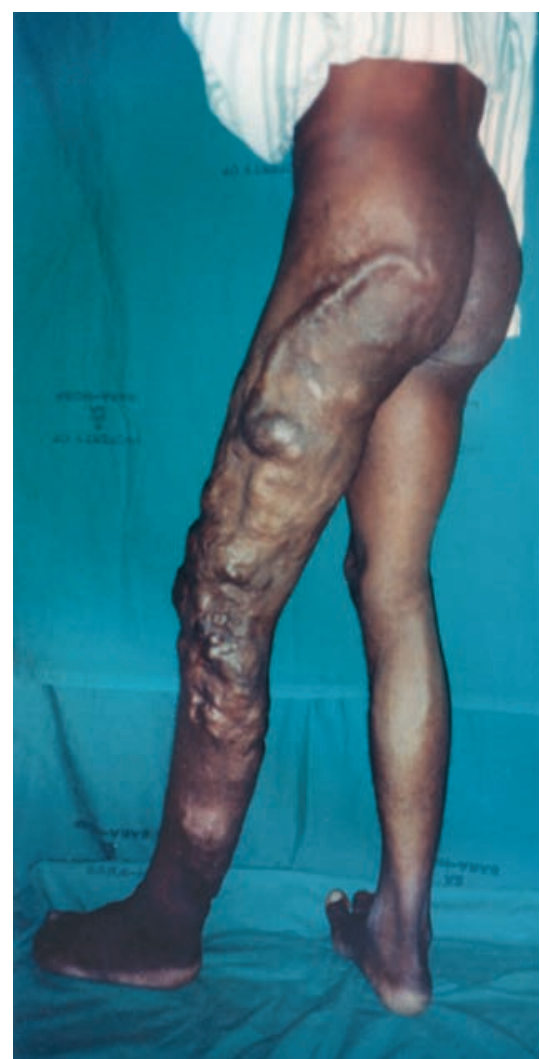

Fig. 1. A patient with severe limb varicosities due to an arteriovenous malformation. These are located superiorly and laterally and not in the vena saphena magna area of distribution. Note also the overgrowth of the affected limb.

in a chronic phase with established varicose veins. It is likely that cases 1 and 2 were due to previous thrombosis. These patients presented in adulthood, and thrombophilias and congenital anatomical abnormalities are therefore unlikely. While thrombosis as the cause of their IVC occlusion could not be proven, another case clearly illustrated this pathology. A 47-year-old man was treated for acute iliofemoral thrombosis. Within a week of admission he developed prominent venous channels over the lower abdomen. Suspected extension of the thrombosis to the IVC was confirmed by both ultrasound and venography. The patient was treated with full anticoagulation.

In case 3, the 12-year-old boy's right groin swelling was understandably mistaken for a hernia, especially because it became larger and painful on performing a Valsalva manoeuvre. The presence of prominent varicosities over the saphenofemoral junction ('saphena varix') is an uncommon condition that must be distinguished from a groin hernia. ${ }^{[1]}$ However, careful clinical examination would have shown extension of the swelling onto the trunk and revealed

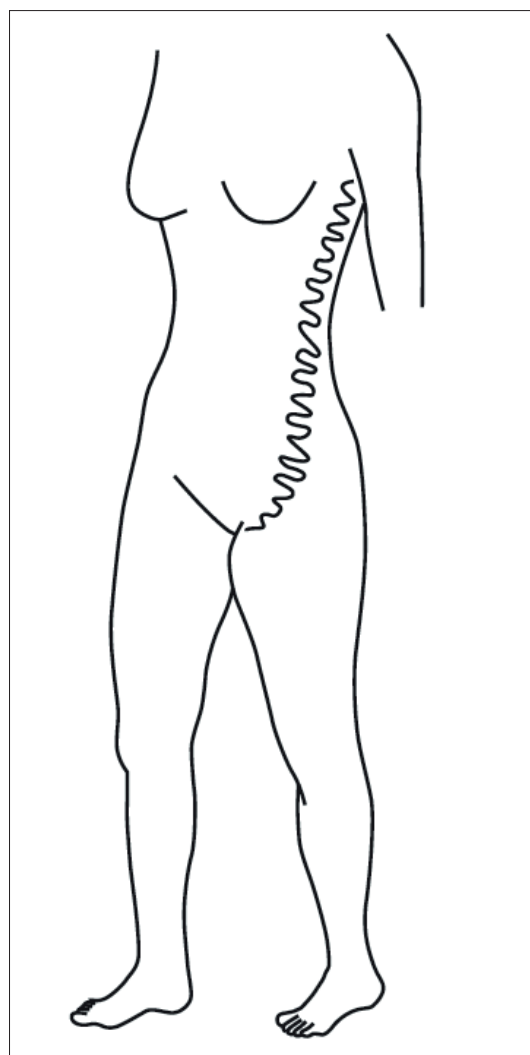

Fig. 2. A sketch illustrating the typical distribution of varicosities on the trunk in case of IVC obstruction. Blood flow is cephalad in such veins.

that the mass consisted of prominent veins. The thrombosis in this case may have resulted from dehydration in earlier childhood, as severe dehydration may lead to IVC thrombosis in children. ${ }^{[4]}$ Iatrogenic thrombosis from IVC cannulation for longterm parenteral nutrition and intravenous infusions has also been reported in this age group.

Isolated IVC thrombosis is sometimes associated with intra-abdominal malignancy, especially renal and pancreatic tumours, and these should be actively excluded. The case of hepatoma described above illustrates such a neoplastic cause. Thrombosis in cancer results from the hypercoagulability associated with malignancy and/or local compression or infiltration of veins. ${ }^{[5]}$ Both these mechanisms were probably operative in this patient.

Case 5 illustrates a cause of limb varicosities that can be confused with extensive occlusion of the deep venous system. AV malformations of the type that contain AV fistulas (Klippel-Trenaunay-Parkes-Weber syndrome) also exhibit extensive venous tortuosities and recurrent varicosities. ${ }^{[6]}$ As 


\section{FORUM}

can be seen in Fig. 1, the varicosities are unusually distributed on the leg (superior and posterior) and there is overgrowth of the affected limb. IVC occlusion should be sought in patients with such varicosities, but other causes such as an AV malformation should be entertained before saphenofemoral vein stripping is done.

Varicosities on the torso should be noted. Prominent veins on the flanks and lateral chest wall could be collaterals caused by IVC obstruction, as depicted in Fig. 2.

It should be noted that two of our patients had their minds set on immediate surgery, as their respective doctors had referred them for varicose vein stripping. Judicious varicose vein surgery can be contemplated for recalcitrant ulceration in such cases. However, cognisance must be taken of saphenous vein tributaries that provide bypass around IVC occlusion, because disruption of these channels can worsen venous hypertension.

\section{Conclusion}

There can be more to varicose veins of the legs than meets the eye. It is important that doctors examine their patients' torsos carefully for prominent veins in all cases of lower limb varicosities and atypical groin hernias. IVC occlusion, or more rare causes such as AV malformations, should be considered in such cases, and appropriate treatment recommendations made. Extra vigilance is called for when a patient has recurrent varicose veins following previous surgery. While referral for directed surgery can be amicably received, this is not always appropriate. ${ }^{[7]}$ Incorrect diagnosis and inappropriate referral can sour relationships between colleagues and with patients.

\footnotetext{
Morris PJ, Malt RA, eds. Oxford Textbook of Surgery. New York: Oxford University Press, 1994:503-

2. Feddock CA. The lost art of clinical skills. Am J Med 2007;120(4):374-378. [http://dx.doi.org/10.1016/j. amjmed.2007.01.023]

3. Oliveria SA, Heneghan MK, Cushman LF, et al. Skin cancer screening by dermatologists, family practitioners, and internists: Barriers and facilitating factors. Arch Dermatol 2011;147(1):39-44. http://dx.doi.org/10.1001/archdermatol.2010.414]

4. Harris RD. The etiology of inferior vena caval obstruction and compression. CRC Crit Rev Radiol Nucl Med 1976;8(1):57-86

5. Young A, Chapman O, Connor C, et al. Thrombosis and cancer. Nat Rev Clin Oncol 2012;9(8):437-49. [http://dx.doi.org/10.1038/nrclinonc.2012.106]

6. Oduber CEU, van der Horst CMAM, Hennekam RCM. Klippel-Trenaunay syndrome: Diagnostic criteria and hypothesis on etiology. Ann Plast Surg 2008;60(2):217-223. [http://dx.doi.org/10.1097/ SAP.0b013e318062abc1]

7. Smith FC, Gwynn BR. Direct access surgery. Ann R Coll Surg Engl 1995;77(2):94-96.
}

Accepted 15 May 2014 